Voix et Images

volxetimages

\title{
Écrire du côté de la mort
}

Michel Biron

Volume 27, numéro 2 (80), hiver 2002

La sociabilité littéraire

URI : https://id.erudit.org/iderudit/290060ar

DOI : https://doi.org/10.7202/290060ar

Aller au sommaire du numéro

Éditeur(s)

Université du Québec à Montréal

\section{ISSN}

0318-9201 (imprimé)

1705-933X (numérique)

Découvrir la revue

Citer cet article

Biron, M. (2002). Écrire du côté de la mort. Voix et Images, 27(2), 337-342.

https://doi.org/10.7202/290060ar d'utilisation que vous pouvez consulter en ligne.

https://apropos.erudit.org/fr/usagers/politique-dutilisation/ 


\title{
Roman
}

\section{Écrire du côté de la mort}

\author{
Michel Biron, Université du Québec à Montréal
}

Le premier roman de Nelly Arcan, Putain ${ }^{1}$, a été reçu avec un tapage médiatique qui vient donner raison à tous ceux qui estiment que la littérature obéit de plus en plus aux règles du vedettariat. Il serait toutefois extrêmement dommage de s'en tenir au seul succès de scandale et de lire Putain en ayant l'impression de mor. dre à un hameçon. Écartons d'emblée les superlatifs: il ne s'agit ni d'un livre génial ni même d'une confession prodigieusement scandaleuse. Il faut être amnésique pour penser que le scandale est quelque chose de radicalement nouveau en littérature. Là n'est pas la force du récit de Nelly Arcan car il y a bien quelque chose de fort ici. L'entreprise ne tient qu'à un fil, mais celui-ci ne se rompt jamais et se dévide au contraire comme si rien ne pouvait le couper. Ce fil, c'est une expérience unique, l'aventure d'une personne si seule qu'on la dirait morte à la société. Cela commence par la haine de soi et de la famille, puis la haine s'étend à ceux et celles que la narratrice côtoie. Celle-ci déteste plus que tout les gens qui lui ressemblent, les clients qui ont son âge, les autres putains, les femmes en général. Elle ne s'en prend pourtant à personne directement et ne cherche ni vengeance, ni réparation, ni guérison. Son réquisitoire n'en a pas la forme: c'est la con- fession gratuite et passionnée d'une putain couchée sur le divan d'un psychanalyste. Malgré ce qui fait l'objet de sa haine, elle n'écrit pas contre la famille, la société ou les hommes en général. Elle écrit comme si elle était ailleurs, de l'autre côté des choses. Voilà ce qui arrive, dira-t-elle à la toute fin du récit, "lorsqu'on interpelle la vie du côté de la mort" (p. 187).

Élevée dans un couvent, la narratrice de Putain ressemble à l'Hélö̈se d'Une saison dans la vie d'Emmanuel de Marie-Claire Blais. Le couvent a l'air plus archaïque encore aujourd'hui, comme s'il sortait d'un temps complètement oublié, figé dans l'histoire et perdu dans les interstices de la géographie (il se trouve quelque part à la lisière de la frontière américaine). La narratrice de Putain se distingue toutefois d'Hélö̈se en ce qu'elle poursuit des études de littérature à l'université. Elle lit Sade: "Et de raconter ces une, deux, trois mille fois où des hommes m'ont prise ne peut se faire que dans la perte et non dans l'accumulation, d'ailleurs vous les connaissez déjă, les cent vingt jours de Sodome, vous les avez lus sans avoir pu tenir jusqu'à la fin, et sachez que moi j'en suis à la cent vingt et unième journée, tout a été fait dans les règles et ça continue toujours, cent vingtdeux, cent vingt-trois." (p. 26-27) Oui, 
ça continue ainsi suivant une logique de la répétition, du ressassement, de l'obsession: ce sont toujours les mêmes gestes mécaniques, les mêmes non-événements, les mêmes mensonges, les mêmes hommes qui défilent, sept ou huit par jour. Et, surtout, ce sont les mêmes souvenirs, "la mère qui larve" et "le père qui jouit". Tous les hommes sont à l'image du père, qu'ils soient ses professeurs, ses clients ou son psychanalyste; toutes les femmes sont à l'image de la mère, éternelles ennemies.

"Et vous devez vous demander pourquoi tout ça alors, pourquoi je ne quitte pas ce commerce que je dénonce et qui me tue, je n'en sais rien [...]." (p. 51) La réponse ne surprend guère: on ne s'attendait pas vraiment à ce que la putain renonce à son personnage. Mais à qui parlet-elle au juste? À qui renvoie ce "vous" qui revient tout au long du récit? Est-ce à son psychanalyste? Au lecteur? Chaque phrase de cette confession semble en tout cas tirée d'un dialogue dont on aurait enlevé systématiquement les répliques ou les questions. La véhémence de Putain, son halètement ("la façon que j'ai de haleter mon histoire comme si j'étais en plein accouplement " [p. 54]), s'explique peut-être d'abord et avant tout par ce procédé qui consiste à apostropher un destinataire absent dans lequel le lecteur est invité à se projeter. "Je n'ai pas l'habitude de m'adresser aux autres lorsque je parle", lit-on dans les pages liminaires. C'est pourtant bien ce qu'elle fera du début à la fin, dès les premiers mots du récit proprement dit: "Oui, la vie m'a traversée, je n'ai pas rêvé, ces hommes, des milliers, dans mon lit, dans ma bouche, je n'ai rien inventé de leur sperme sur moi, sur ma figure, dans mes yeux, j'ai tout vu et ça continue [...]." (p. 19) Mais à qui cette femme dit-elle "oui"? Et à qui cette femme semble-t-elle dire: suivez mon regard, si ce n'est au lecteur qu'elle invite à voir ce qu'elle a vu? Putain n'est pas seulement un récit autobiographique ou une autofiction: Putain, c'est nous-même.

La putain de Nelly Arcan n'a pas besoin de faire de la sollicitation pour attirer des clients: elle les attend dans une chambre louée par l'agence d'escortes qui l'emploie. Elle ne racole pas les hommes, pas plus que le récit ne cherche à séduire son lecteur. D'une certaine manière, elle n'existe que si nous allons vers elle. Sans le regard du voyeur, elle n'est rien qu'une image toute faite, une fragile abstraction, une énigme résolue d'avance. Même sa haine ne lui appartient pas en propre: elle remonte à la nuit des temps et demeure semblable à ce qu'on attend d'elle depuis toujours. Son nom propre n'existe pas davantage: elle n'a qu'un prénom, inventé pour les besoins de la cause (Cynthia, prénom de sa sœur morte dans la prime enfance). Son corps enfin a quelque chose d'irréel: il n'est qu'une coquille vide dans laquelle chaque homme projette d'autres images de femmes. Nulle identification possible avec Cynthia, puisqu'elle joue à n'être qu'une image qui porte un "nom de putain".

Il est assez paradoxal que l'autofiction, genre par excellence de l'authentique, de l'intime, rabatte la singularité du moi sur une image déjà là, sur un prénom qui n'est rien de plus qu'une raison sociale. On sort cependant de la lecture de Putain avec l'impression que tout est vrai, que le personnage a véritablement intériorisé ce 
cliché, que le moi ne cherche plus à préserver de quelque façon sa conscience contre l'image que les autres lui renvoient. Je suis telle que vous me voyez, je n'en démordrai pas, c'est vous qui rêvez, c'est vous qui vous racontez des histoires si vous pensez que je suis une autre. Ici, c'est la vérité la plus nue, le spectacle d'un corps qui ne prétend plus à aucun secret. Ici, il n'y a plus d'arrière-scène, de zone invisible: entre le privé et le public, entre l'intime et le mondain, il n'y a plus de mur qui tienne. L'obscène abolit tout mystère.

La force du récit, dont je parlais au début de cette chronique, n'a rien de rassurant pour l'avenir du roman. Les avatars récents de l'autobiographie font peser sur le roman une menace sans précédent. Comme le constate l'un des plus ardents défenseurs du genre, Lakis Proguidis: "Cette dernière menace de l'autobiographisation est d'ailleurs la seule qui puisse être fatale pour l'art du roman, car c'est la seule qui n'affronte pas directement son ennemi mais corrode son âme ${ }^{2}$." L'autofiction a beau se présenter chez Nelly Arcan comme un récit, et non comme une autobiographie, il n'en reste pas moins qu'elle joue sur l'identification partielle entre les figures de la narratrice et de l'auteure. Le personnage n'est pas tout à fait un personnage: c'est là une ambiguité qu'il sera difficile de lever. On peut en jouer, comme le fait par exemple la romancière Christine Angot, mais à condition de ne pas oublier que le loup est dans la bergerie.

\section{**}

"Dieu hait son âme. Et puis fuck $^{3}$." Ainsi se termine $D u$ mercure sous la langue du romancier Sylvain Trudel, qui raconte les dernières semaines de son personnage, Frédéric Langlois, à l'aube de ses dix-sept ans. Atteint d'un cancer à la hanche, il sait qu'il ne sortira plus de l'hôpital et il écrit, à la manière des romantiques, une sorte de lettre d'adieu. Mais c'est un romantique froid, glacial, "crinqué" (p. 8) à mort, à la limite du cynisme et de la misanthropie. Il a l'âge des poètes de génie et se prend d'ailleurs pour un poète. Son nom de plume, "Métastase", donne une idée du type de personnage. Comme Nelly Arcan, Sylvain Trudel écrit du côté de la mort, une mort imminente qu'il ne souhaite d'aucune façon éviter ou adoucir. Même si le miracle de sa guérison se produisait, crâne-t-il, il le refuserait et se suiciderait sans hésiter. Personne ne veut croire qu'un garçon aussi gentil se ferme obstinément aux discours de consolation. Ni sa famille, ni les infirmières, ni la psychothérapeute, ni le prêtre ne comprennent que la mort soit déjà son royaume.

Or, ce royaume fictif de la mort, le personnage romanesque de Frédéric Langlois n'en a plus du tout le monopole aujourd'hui. Des personnages appartenant à d'autres genres (que ce soit le témoignage d'écrivains morts du sida comme Hervé Guibert ou des autofictions comme celle de Nelly Arcan) lui volent la vedette. On voudrait opposer à une telle concurrence la puissance d'imagination propre au roman, mais Du mercure sous la langue, en se plaçant du côté du témoignage fictif, joue sur le terrain de l'adversaire. On lit le discours de Frédéric Langlois comme on regarde les tours de magie d'un prestidigitateur à la télévision: le téléspectateur veut y croire, 
mais il y croirait davantage s'il n'y avait pas la magie de la télévision qui venait se superposer à la magie du geste.

Cela dit, Du mercure sous la langue n'a rien d'artificiel ou de fabriqué. Dans une langue drôle et acide à la fois, Trudel prend la température du monde actuel, depuis ce purgatoire qu'est l'hôpital. Frédéric y rencontre d'autres adolescents gravement malades et tous ensemble forment une sorte de société des poètes sur le point de disparaître. Parmi celle-ci, il y a surtout Benoît, spécialiste de la Bible, et Marilou, qui écrit les plus beaux poèmes et à qui il aurait voulu faire un enfant. Il y a aussi la grand-mère, la seule personne de la famille devant qui Frédéric avoue ses désirs de poète et ses peurs de petit garçon à moitié mort. À part ces complices qui côtoient la mort de près, il n'y a que des ombres de vivants, de "pôvres zenfants" (p. 103) qui refusent de voir la réalité en face, et surtout ce Dieu que Frédéric déteste de toute sa force, avec une impiété vindicative. Du mercure sous la langue est porté de bout en bout par cette haine de Dieu et par un rejet définitif et héroïque du monde.

Depuis Ducharme, aucun écrivain n'a démontré autant de talent que Trudel pour exprimer la violence la plus radicale à travers les propos faussement naifs d'un enfant. Du mercure sous la langue ne fait pas exception dans son cuvre: on y retrouve une langue à la fois chaleureuse et crue, pleine d'humour mais aussi d'une angoisse sourde, sans éclat. Il est toutefois plus près que jamais de la colère mystique et il s'attaque directement aux textes sacrés, notamment aux épîtres de Saint-Paul dont il raille le mauvais génie: "Où est-elle, ô mort, ta victoire?" (p. 94) Frédéric
Langlois ne veut même pas entendre les discours consolateurs des intermédiaires mous que sont le prêtre ou la psychothérapeute: il remonte à la source, à ce que le critique torontois Northrop Frye appelait le grand code. Il n'en espère aucune rédemption et il n'a aucune velléité de conversion tardive, mais, au moins, il y rencontre des adversaires de sa taille.

\section{$* *$}

Les espaces secrets ${ }^{4}$ de Francine Prévost est un petit livre discret, écrit avec une retenue qui rappelle le ton intimiste de romancières comme Louise Dupré. La violence des sentiments y est toutefois beaucoup plus crue que chez cette dernière, à peine moins forte que dans Putain. La narratrice s'appelle Éva, du nom de sa sœur mort-née, et se décrit comme une ancienne mystique. Plus jeune, elle lisait Thérèse de Lisieux et possédait tout un arsenal de méthodes de mortification. Puis elle se marie, quitte son époux pour se réfugier à Paris, où la rejoint son frère avec lequel elle entretenait depuis longtemps une relation incestueuse. Le frère en question se jette dans la Seine, la laissant plus seule que jamais. Elle se rend alors en Suisse pour terminer un roman chez un couple d'amis, Günther et Marthe.

Les espaces secrets prend la forme d'une lettre adressée à Günther, suivie de la confession d'Éva. Elle y parle de lamour inavouable quelle a rapidement éprouvé pour cet homme "façonné par la lumière de la montagne" (p. 26), et de sa jalousie de femme stérile devant le ventre grossissant de Marthe. Elle raconte comment elle a trahi la confiance de celle-ci, au 
prix d'une méchanceté qui lui était étrangement facile. Elle décide pourtant de s'éloigner de Günther et s'installe alors à Fribourg (elle ne veut surtout pas revenir à Montréal). De là, elle reçoit une invitation de Günther, mais c'est à Marthe qu'elle veut d'abord parler, comme s'il lui fallait d'abord'se vider le cœur, tout dire, tout avouer, rompre le silence, succomber à un type de désir tout aussi brutal que la pulsion charnelle, ce qu'elle nomme un "désir d'intimité" (p. 58). Dans le même mouvement, elle se découvre de nouvelles réserves de rage et se met à écrire plus crûment que jamais auparavant, en s'adressant directement à sa sœur:

\begin{abstract}
Étonne-moi, surprends-moi, invente-moi une vie. Fais de moi ta putain, ton trésor, ton objet de jouissance. Glisse ta main sous mes reins. Marque-moi au fer rouge comme si j'étais à toi, comme si j'étais ta propre douleur que tu entretiens. Je suis ta douleur, je stis ton manque, je suis le trou qui t'a vue natitre, la membrane qui recouvre ton sexe. Je suis ta soeur et tu es mon inage et je serai maudite pour t'avoir dit mon amour. (p. 61)
\end{abstract}

La blessure la plus profonde du personnage ne se joue donc pas autour de la relation maternelle, mais dans le souvenir obsédant de la perte de la sœur. La figure mythique de référence, c'est Cainn tuant son frère Abel, et non CEdipe ou Jocaste. La narratrice reprend les choses à partir de là. Elle intitule son propre roman Le livre de Caïn et célèbre comme une victoire l'ultime changement de point de vue: "Je l'ai entièrement réécrit du point de vue de Cain et non plus du point de vue d'Ève." (p. 67) Façon de dire que ce roman très féminin refuse d'appliquer à la lettre la leçon de la psychanalyse. Il ne croit pas aux récits tout faits et n'hésite pas à emprunter la voix de l'autre, celle du frère meurtrier, pour faire entendre sa propre énigme. C'est là l'aspect le plus original et le plus troublant de ces Espaces secrets.

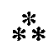

Le joueur de flûte ${ }^{5}$, sixième roman de Louis Hamelin, raconte la quête de Ti-Luc Blouin, parti dans l'Ouest canadien à la recherche de son père. Spécialiste du "fuck writing", technique d'écriture qui consiste à faire asseoir une femme sur le clavier d'une machine à écrire, ce père s'appelle tantôt Forward Fuse, tantôt Mister Big, et il joue de la flûte. Ancien héros de la contre-culture californienne, cet écrivain américain est devenu une sorte de légende (plus ou moins vivante) réfugiée dans une ancienne commune. Le narrateur n'a plus que cet homme pour se rattacher à son passé, à lui-même. Sa dernière blonde vient de le quitter pour manque de caractère. Il est habitué: "Ce n'est pas pour me vanter, mais je suis seul au monde." (p. 17) Il se présente fièrement comme un jeune homme faible à tous points de vue, même physiquement. Il souffre en effet d'un problème à la colonne vertébrale et risque de s'écrouler à tout moment. En un seul paragraphe particulièrement réussi, il brosse son autoportrait:
Attention, fragile. Je fais un mètre soixante avec des talons. Très tôt, iaj appris à bomber le torse pour paraître plus grand. Mais ça ne suffit pas toujours. Alors on s'ar- range. On se moule. Je suis un homme sans goût. Au restaurant, si on me dit que le vin est bon, je tombe d'accord à tout coup, mais 
jamais je n'aurais été capable de reconnaître le fait par moi-même. Je modèle mon jugement sur celui des autres et me méfie de l'expérience de mes propres sens. Avoir des goûts affirmés est quelque chose qui me fatigue. (p. 17)

Le bon vin, Ti-Luc Blouin n'aura pas souvent l'occasion d'en goûter dans les forêts de la ColombieBritannique, où il espère retrouver la trace de son père. Il y boit plutôt du thé noir préparé par Gros-Max, l'un des militants venus barrer la route aux bûcherons de la compagnie Westop, par ailleurs ancien éditeur d'un bulletin montréalais d'obédience lacanienne (organe de la Ligue des associations anarcho-libidinales d'Amérique, la LAALA). Il rencontre aussi une fille, Muse, et son rat (Raymond), flanquée d'un peintre du Plateau Mont-Royal, Arnot Valanti. Le couple s'était rencontré aux Foufounes électriques: Muse faisait de la "strip poésie" pendant que l'autre se dépêchait de peindre son corps. On rencontre encore d'autres énergumènes dans le campement de fortune construit pour les besoins de la cause, notamment autour d'un sauna artisanal: on y voit, tous aussi nus les uns que les autres, un ancien activiste de Greenpeace appelé Paul Watchcock, un correspondant de Libération au nom surcomposé (Pierreau Marchal-Dubond-Dubont), une Allemande "sculpturale" et ainsi de suite.

La quête de Ti-Luc rappelle la traversée de l'Amérique de Jack Waterman dans Volkswagen Blues de Jacques Poulin. On se souvient que le héros de Jacques Poulin tentait de retrouver son frère et qu'il mettait ses pas dans ceux des premiers explorateurs du continent. Dans Le joueur de flûte toutefois, ce n'est pas le voyage qui compte: c'est le lieu d'arrivée et la faune hétérogène que le narrateur y découvre. Son enquête le conduit directement au but, grâce aux témoignages d'anciens compagnons d'armes de son père. Aucune énigme digne de ce nom ici, nous savons rapidement où se trouve la commune de Mister Big, la bien nommée "Love Mountain". Privé du plaisir de la quête, le lecteur se trouve tout à coup devant l'homme en question. Il apprend à connaître le père, mais le personnage est si peu intéressant qu'il ennuie. C'est comme si on avait abandonné en cours de route le héros de départ, l'homme fragile, au profit d'un joueur de flûte qui appartient à une autre histoire. Comme si Jacques Poulin avait commencé à raconter la vie de Théo, le frère paralysé de Jack Waterman. Or, Mister Big n'est pas un véritable personnage, pas plus que Jack Waterman. C'est un has been, le symbole d'une Amérique révolue, illusoire, survivant de peine et de misère grâce à des cachets de codéine. On est un peu soulagé quand il finit par se tirer une balle dans le crâne, mais c'est trop tard: le mal est fait. Le roman ne peut plus retrouver son équilibre et la fin verse dans une symbolique lourde et confuse. C'est dommage, car Le joueur de flute avait commencé en beauté, porté par une écriture qui s'est étonnamment raffermie depuis La rage ${ }^{6}$.

1. Nelly Arcan, Putain, Paris, Seuil, 2001, $187 \mathrm{p}$.

2. Lakis Proguidis, De l'autre côtê du brouillard Essai sur le roman français contemporain, Québec, Nota bene, 2001, p. 162-163.

3. Sylvain Trudel, Du mercure sous la langue, Montréal, Les Allusifs, 2001, p. 130.

4. Francine Prévost, Les espaces secrets, Montréal, Québec Amérique, 2001, 69 p.

5. Louis Hamelin, Le joueur de flâte, Montréal, Boréal, 2001, 227 p.

6. Louis Hamelin, La rage, Montréal, Québec Amérıque, $1989,405 \mathrm{p}$. 